\title{
Cacodémon cinéaste (à propos de Looking for Richard)
}

Jean-Michel Déprats

\section{OpenEdition}

Édition électronique

URL : http://journals.openedition.org/shakespeare/222

DOI : 10.4000/shakespeare.222

ISSN : 2271-6424

Éditeur

Société Française Shakespeare

Édition imprimée

Date de publication : 1 novembre 1998

Pagination : 91-103

ISBN : 2-84269-230-6

\section{Référence électronique}

Jean-Michel Déprats, "Cacodémon cinéaste (à propos de Looking for Richard) », Actes des congrès de la Société française Shakespeare [En ligne], 16 | 1998, mis en ligne le 01 novembre 2007, consulté le 02 mai 2019. URL : http://journals.openedition.org/shakespeare/222 ; DOI : 10.4000/shakespeare.222 


\section{S H A K E S P E A R E \\ \& $\quad$ L E $\quad$ C I N É M A}

Société Française Shakespeare

Actes du Congrès de 1998

米 $*$ *

Textes réunis et présentés par

Patricia DORVAL

publiés sous la direction de

Jean-Marie MAGUIN 
Site web : <http : //alor.univ-montp3.fr/serinf/SFS/> Liste de diffusion : <sfs-1@smrl.univ-montp3.fr >

Tous droits de traduction, de reproduction et d'adaptation réservés pour tous les pays.

(C) 1998. Société Française Shakespeare,

École Normale Supérieure, 45 rue d'Ulm. 75005 Paris.

ISBN 2-84269-230-6 


\section{A C O D ÉMON C IN É A T T}

Ces toutes dernières années ont vu s'épanouir sur nos écrans une floraison remarquable de films «shakespeariens» : il y a eu l'Othello d'Olivier Parker avec Kenneth Branagh, Laurence Fishburne et Irène Jacob, le Richard III en uniformes fascistes de Richard Loncraine avec Ian McKellen, Annette Bening et Kristin Scott-Thomas, le Roméo et Juliette chromo et kitsch de l'Australien Baz Luhrmann avec Claire Danes et Leonardi DiCaprio, une Nuit des Rois sensuelle et sensible mais peu cinématographique signéc Trevor Nunn, et le grand œuvre de Kenneth Branagh, ce Hamlet en version longue et courte aussi décevant qu'attachant qui a attiré en France 150000 spectatcurs '. Au sein de cette abondante production cinématographique qui fait de Shakespeare le scénariste le plus populaire d'Hollywood, il y a tout lieu de marquer d'une pierre blanche le surgissement d'un ovni cinématographique ${ }^{2}$, le Looking for Richard ${ }^{3}$ d'Al Pacino auquel je voudrais rendre hommage. Mon propos est seulement d'évoquer cette œuvre, de tenter d'en dégager la singularité et l'originalité, d'en souligner l'intérêt et les enjcux. Assurément, il y a quelque inconvenance de ma part à prendre en otage un auditoire de votre qualité et de votre science dans le seul but de vous communiquer mon enthousiasme pour ce film. J'ai bien conscience de ne pas vous présenter un travail de recherche, et vous me pardonnerez peut-être de ne pas me livrer à l'une de ces savantes analyses sémiologiques de la représentation filmique qui font le charme discret mais parfois délétère de la pensée hexagonale en ce domaine.

De Richard III, malgré la popularité constante de la pièce au théâtre ${ }^{4}$, il n'y a eu à ce jour que deux réalisations cinématographiques majeures : le film pionnier de Laurence Olivier (1955) avec, outre Laurence Olivier dans le rôle- 
titre, Claire Bloom (Lady Ann), John Gielgud (Clarence), Ralph Richardson (Buckingham), et la version déjà citée de Richard Loncraine (1986), remake cinćmatographique d'un grand succès de théâtre. A revoir aujourd'hui le film de Laurence Olivier, on s'étonne qu'il puisse passer pour un parangon de fidélité shakespearienne: le rôle de Margaret est coupé, le générique annonce : "Laurence Olivier presents Richard III by William Shakespeare with some interpolations by David Garrick and Colley Cibber, etc.» et de fait, le monologue initial est tronçonné, flanqué d'extraits d'Henry VI, il reste peu de choses de la scène avec Lady Ann, déplacée dans la chronologie de la pièce. Ce Richard III reste du théâtre filmé avec décors et costumes "médiévaux», très «Riches heures du duc de Berry», fanfares de cuivre et oriflammes. L'interprétation a vieilli : Laurence Olivier a une diction sèche, un jeu un peu monochrome et guindé, dénué d'humour ${ }^{5}$, Claire Bloom a la voix chevrotante et le regard égaré, Giclgud, hiératique, y abuse de son talentueux vibrato et le film manque de rythme. On ne peut pas faire le même reproche au film de Richard Loncraine qui, transposant l'action dans une Angleterre imaginaire des années 30 gangrenée par le nazisme, signe un Richard III new look, échevelé, dont la fin dérape dans le film catastrophe mais qui bénéficie de l'interprétation magistrale de Ian McKellen. Lequel campe, non sans cabotinage parfois, un Hitler british mâtiné de loup de Tex Avery. Dix ans avant le film de Richard Loncraine, Raoul Ruiz avait réalisé, lui aussi d'après une mise en scène de théâtre, un étonnant Richard III énigmatique et baroque qui n'a jamais été distribué. Ruiz a inventé une fiction avec les comédiens de Georges Lavaudant et les mots de Shakespeare. Décors : un lac de montagne, les souterrains d'une forteresse, une grotte de studio... Il plonge la tragédie dans un univers pictural et musical baroque, et chaque image est un hommage à la pcinture. Ruiz n'a pas filmé le théâtre sur scène, ni conçu un film de cape ct d'épéc. Richard III s'est mué en une fiction ruizienne : tantôt film d'aventure, tantôt parodie d'une tragédie sanglante. La musique amplifie en tempête l'épopée romantique de ce roi de théâtre interprété par Ariel GarciaValdès qui compose un monarque infantile et dangereux, archange blond du crime. Ce n'est plus du Shakespeare... mais c'est un vrai film. Eternel dilemme!

Aux antipodes de ces réalisations qui restent fondées sur le texte shakespearien, Looking for Richard n'est pas une version cinématographique de plus de Richard III. C'est un génial bricolage et un film gigogne où s'enchassent plusicurs films potentiels. Al Pacino a tourné Looking for Richard sur une période de quatre ans, occupant les espaces libres entre ses tournages de Heat ou de City Hall, jonglant avec les emplois du temps de ses camarades comédiens. «Au début, dit-il, c'était une sorte d'expérience... Je ne pensais pas du tout que ce projet finirait pas être présenté au Festival de Cannes» ${ }^{6}$. Il devait être projeté dans les collèges et les lycées. Preuve de 
l'aspect expérimental et de la liberté économique du projet, Pacino a imprimé quatre-vingts heures de pellicule avant d'en arriver aux deux heures du résultat final. Montrer les préparatifs et les répétitions de la pièce, inclure le processus de création dans le film lui-même permettait à Pacino de «desceller le monument» mais aussi de montrer au public le long, patient et difficile travail de l'acteur.

D'entrée de jeu, le générique corrige King Richard en Looking for Richard indiquant par là que le spectateur ne va pas voir une mise en scène complète et définitive de Richard III mais un chemin, une quête, un travail préparatoire pour arriver à la pièce. «Quand je faisais des lectures dans les universités, rapporte Pacino, je me souviens qu'en expliquant d'abord les intrigues et les personnages, dans un langage simple et direct, tout cela avant d'entrer spécifiquement dans Shakespeare, les étudiants devenaient beaucoup plus réceptifs. On a repris cette méthode dans le film» ${ }^{7}$. Ainsi, Looking for Richard se présente sous la forme d'un documentaire-fiction sur une troupe qui répète Richard III. C'est un brillant essai cinématographique sur l'actualité de Shakespeare, les rapports du théâtre et du cinéma, l'art de l'acteur, le travail d'un rôle, la relation - problématique - des Américains à Shakespeare... Tour à tour documentaire, journal de répétitions, work in progress, (xuvre de fiction, ce film-puzzle, «formule hybride, sinon difforme, comme son héros», note Serge Chauvin ${ }^{8}$, entrelace plusieurs manières : extraits de Richard III (les grandes scènes), interviews de gens de la rue et de spécialistes universitaires et gens de théâtre - , séances de travail à la table, visites à Stratford et à Londres, etc. Il mène l'enquête avec passion, avec une ćnergic, une vitalité, une curiosité et une intelligence de chaque instant dont témoignent le montage haletant et les tressautements de la caméra qui serre les visages au plus près. «C'est un film, dit Al Pacino, qui ressemble à ce que peut être le métier de comédien, la façon dont on peut travailler un rôle comme une enquête" ${ }^{9}$. C'est du cinéma d'esquisse, de recherche et d'approfondissement mené tambour battant. Du cinéma pédagogique et ludique. Sérieux et rigolard. Sans une once de lourdeur, d'académisme ou de solennité. En costume de ville ou en costume de scène, caméra au poing, à sa table de travail ou sur un plateau de théâtre, Al Pacino interroge, s'interroge, ausculte, dissèque, cabotine, s'esclaffe, en synergie et dans une osmose étonnante avec le fabuleux histrion du crime que Shakespeare a créć et à la recherche duquel l'interprète de Coppola, Schatzberg, De Palma, se lance une nouvelle fois avec fougue après avoir joué le rôle à deux reprises au théâtre (Boston et New York).

Le joyeux désordre qui préside à cette exploration bouillonnante - et dont le montage fébrile accentue le tourbillon - empile et brasse plusieurs plans d'enquête et de réflexion liés entre eux de façon logique et successive comme autant d'étapes préparatoires à l'interprétation : 
- scènes de rue et micro-trottoir destinés à sonder les rapports ambigus des Américains à Shakespeare.

- sćances de travail à la table animées par le questionnement des acteurs sur les motivations, personnelles et politiques, de leurs personnages.

- pélerinage sur les lieux mythiques de la légitimité shakespearienne (maison natale de Shakespeare à Stratford, théâtre du Globe en reconstruction).

- commentaires érudits des universitaires (principalement Emrys Jones et Barbara Everett) sur le contexte historique, la guerre des deux roses, les clans en présence.

- mises en perspective des acteurs britanniques (Kenneth Branagh, Derck Jacobi, John Gielgud, Vanessa Redgrave) et des metteurs en scène (Kenneth Branagh, Peter Brook) sur les modes d'élocution du texte, la scansion du vers, etc.

- répétitions et interprétation des scènes (en costumes de ville puis en costumes de scène) à partir des hypothèses de travail examinées au cours des étapes précédentes.

Le film avance en spirale en parcourant tous ces cercles concentriques. A cet étagement vertical s'articule une structure horizontale : le film progresse chronologiquement à travers les scènes majeures de la pièce, du monologue initial à la bataille de Bosworth, acheminant irrémédiablement le spectateur vers la mort de Richard - fin du personnage, apothéose de l'acteur. Le chemin est balisé par une quinzaine d'intertitres qui ponctuent les étapes de la recherche autant qu'ils scandent les phases de la carrière de Richard, ascension ct chute ${ }^{10}$. Le mouvement du film est happé par sa fin. Comme l'écrit Serge Chauvin, «le texte se fait impérieux au point d'infléchir le projet même du lilm [... I. On perd peu à peu la visée de vulgarisation, la réflexion [...] sur le rapport du public à la culture, et même les travaux d'approche proprement dramatique sont phagocytés par l'irréversible progression de la pièce qui devient comme le destin du film»" ". Et si le film s'achève comme il a commencé par la tirade de Prospéro sur l'insubstantialité du monde ${ }^{12}$, cette circularité $n$ 'a rien d'artificiel car les mots qui dissipent l'illusion et révoquent les sortilèges se sont lestés chemin faisant d'une vérité expérimentale. Les lignes de la fiction et du réel se sont croisées à plusieurs reprises. Le réel a nourri la fiction qui, à son tour, a contaminé le réel. Les frontières se sont dissoutes. Richard / Al Pacino en casquette de base-ball ironise sur la fragilité de Lady Ann / Winona Ryder et sur la victoire d'Al Pacino / Richard en pourpoint aux manches parementées. Scènes de répétitions en habit d'aujourd'hui et scènes en costumes d'époque se fondent l'une dans l'autre au montage. Le fils d'un des constructeurs du Globe rencontré sur le chantier réapparaît au plan suivant en enfant d'Édouard, les acteurs parlent de leur personnage à la première personne, Gloucester / Al Pacino agonise dans les 
bras de son metteur en scène el ami Frederic Kimball (qui joue l'Évêque d'Ely dans la scène des fraises), etc.

Par ces va-et-vient, le réalisateur ne cherche pas pour autant à entretenir la confusion entre l'ici-et-maintenant et l'ailleurs-autrefois ou à établir des analogies historiques visant à montrer l'actualité ou la modernité de Shakespeare. Si pour aborder la scène de l'arrestation d'Hastings, un des acteurs suggère un parallèle entre la tenue du Conseil du Royaume ct une réunion des chefs de la Mafia (a gathering of dons), ce n'est qu'une analogie féconde pour que les comédiens imaginent l'atmosphère lourde et l'ironie dramatique de la scène. Al Pacino ne propose pas un Richard III mafioso, quel que soit le nombre de caïds de la pègre qu'il a interprétés au cinćma. Contrairement au film de Loncraine, le film d'Al Pacino ne joue pas le jeu des correspondances. Il insiste sur la distance, la nécessité de reconstituer l'écheveau des donnécs politico-historiques du $\mathrm{XV}^{\mathrm{e}}$ siècle anglais.

Ce qui n'empêche pas le réalisateur de vouloir communiquer sa conviction que le théâtre de Shakespeare, si l'on facilite le chemin vers lui, est bien une dramaturgie pour notre temps. Car la préoccupation d'Al Pacino n'est pas seulement de comprendre et d'interpréter mais de faire comprendre et de faire partager. Il y a toute une dimension pédagogique et prosélytique dans son entreprise généreuse et passionnée. La casquette en visière visséc sur la nuque, Pacino arpente les rues de New York avec Frederic Kimball et interroge les passants. Les réactions oscillent entre fascination et fraycur mais le public américain s'avoue majoritairement réfractaire. «Shakespcare?», «Ennuyeux !», «Compliqué !», «On s'y perd dans les généalogies !», «Je suis parti à l'entracte», «J'ai craqué !» fusent les commentaires des gens dans la rue qui reconnaissent que «Ah oui ! Shakespeare? To be or not to be !» mais avouent le plus souvent qu'ils n'ont pas lu, pas vu ou suggèrent d'un haussement d'épaule que ce n'est pas pour eux. «Si !» rétorque dans un large sourire un sympathique Noir édenté, mi-philosophe, mi-clochard céleste, «Shakespeare apprend à mieux sentir... Les mots aujourd'hui sont dévalués parce qu'il n'y a plus de sentiments vrais [je résume]». Ce rapide microtrottoir effectué dans Manhattan jusque dans Central Park où se déroulc un festival Shakespeare gratuit confirme que l'Amérique résiste à Shakespeare. Respect et dévotion de façade mais découragement du public tenu à distance par les références historiques obscures, la langue archaïque, la teneur constamment métaphorique et poétique du texte. On ne résiste pas au plaisir de citer le commentaire d'un acteur qui se moque gentiment du style shakespearien : "Shakespeare uses a lot of fancy words». Au lieu de dire : «Hey you, get that thing there and bring it back to me» Shakespeare dira : «Be a Mercury, set feathers to thy heels and fly like thought from there to me again !» Autre facteur de résistance ou de rejet, les pesantes conventions d'un style de jeu théâtral à l'extrême, grommelé dans les graves («Oh yes my lord, 
thank you my lord, I shall obey my lord») qu'imite avec humour un autre comédien de la troupe. Les acteurs américains sont intimidés et inhibés par Shakespeare, assure Peter Brook. Qui s'en étonnerait quand le sentiment très britannique de propriété exclusive s'affiche avec morgue. "What the fuck do you know about Shakespeare ?» demande agressivement à Al Pacino le jardinier de la maison natale de Shakespeare à Stratford. «Perhaps the American don't read books or go to museums as much as we do", laisse tomber, hautain, l'ineffable Gielgud, plus british que nature. Et pourtant Shakespeare hante l'Amérique. Se posant la question du décor, plutôt que dans un théâtre, Al Pacino a choisi d'aller filmer au musée des Cloîtres de New York. Qui abrite, on le sait, une église et trois cloîtres romans démontés pièce à pièce en France et en Espagne et reconstruits tels quels au nord de Manhattan. Décor «authentique» donc mais qui est le fruit d'un pillage et d'un transfert. Et qui traduit bien l'ambiguïté du rapport de l'Amérique à l'Europe, ct des Américains à Shakespeare, où se mêlent respect et culpabilité.

Problème de légitimité donc. Al Pacino endosse les habits de l'usurpatcur Richard pour tenter d'établir ses lettres de noblesse théâtrale. Non sans s'être au préalable rendu sur les lieux saints, dont il attend, on le devine, une inspiration illusoire. La visite à la maison natale de Shakespeare ne déclenche qu'une sonnerie d'alarme, les pompiers accourent en catastrophe pour déloger l'intrus. L'épiphanie espérée sur les planches du Globe reconstruit ne se produit pas davantage. Pacino se moque avec humour des mythes (culturels et touristiques) des origines. Tout comme il se moque des fausses certitudes, des théories fumeuses qui prétendent détenir la clé de l'interprétation juste : une brève scène de soirée mondaine suffit à ridiculiser ccux qui tentent d'expliquer Shakespeare par le Talmud ou le yin et le yang, ceux qui s'extasient devant un Macbeth rock and roll ou cherchent à toute force à rapprocher Shakespeare de nous en affirmant (je cite) qu' «Hamlet llippe comme tous les gosses de son âge».

Il n'est pas surprenant que dans sa quête de références et de modèles, Pacino butc sur la question du pentamètre iambique. La connaissance des principes de scansion est-elle le sésame du jeu shakespearien ? La séquence est réjouissante. Devant un Al Pacino médusé, Frederic Kimball explique qu'un iambe est comme un tamanoir ou un fourmilier, court sur pattes devant et très haut sur ses pattes de derrière. Pacino, cabotin, fait des yeux de merlan frit à la caméra. La question posée est de savoir si la diction poétique (verse speaking) est une clé de l'interprétation shakespearienne. Dans le documentaire que Claude Mouriéras a réalisé pour Arte ${ }^{13}$, Fiona Shaw compare le rythme iambique au rythme cardiaque, et souligne avec pertinence la racine physiologique, organique, de la prosodie du blank verse. Dans le film d'Al Pacino, c'est à Vanessa Redgrave qu'il revient de rappeler la base émotionnelle du jeu de l'acteur et d'inverser les rapports d'interdépendance 
supposés du jeu et de la prosodie. Son intervention, dont on s'est gaussé à tort parce qu'elle parle du "pentamètre de l'âme» ${ }^{14}$, mérite d'être citée in extenso: «Shakespeare's poetry and his iambics floated and descended through the pentameter of the soul. And it is the soul, the spirit of real concrete people going through hell and sometimes through moments of great achievement and joy, that's the pentameter you have to concentrate on. And should you find that reality, all these iambics will fall into place». Ce n'est pas à travers la diction juste que l'acteur trouve les états de son personnage mais c'est lorsqu'il atteint la base émotionnelle juste que la prosodic se met en place de manière adéquate et expressive. Cette prise de position ne clôt pas le débat mais elle a au moins le mérite de l'ouvrir. Sur la part d'artifice ou de naturel qu'il convient de donner à la diction du vers, on sait qu'il y a deux écoles. Si l'on transpose en termes français, il y a, d'un côté, l'école Planchon où l'on s'efforce, non de dire le vers comme de la prose, mais en tout cas de trouver d'abord l'authenticité de la parole ou la souplesse de l'élocution, et, de l'autre, avec des variantes, l'école Vitez ou Rist ou Villégier qui donne à entendre nettement la cadence et la structure de l'alexandrin, surprononce les diérèses et les «e» muets. Sans prétendre trancher le débat, je dirai sculement que l'option formaliste a parfois ceci de dogmatique et de mécaniste qu'elle postule que l'émotion juste, l'interprétation sensible découleront inévitablement d'une diction juste.

On pourra trouver ces questions trop techniques mais elles font partic des interrogations et du travail de l'acteur. L'intérêt majeur du film, pour qui n'cst pas familier des répétitions au théâtre, est de montrer le processus d'élaboration du travail théâtral, de donner une image concrète du type de questionnement, aux antipodes de l'approche universitaire ou du commentaire stylistique, qui permet à l'acteur de nourrir son imaginaire. La construction d'un rôle et des rapports des personnages entre eux part de questions concrètes sur le pourquoi et le comment. Pourquoi Lady Ann se jette-t-elle dans les bras de l'assassin de son ćpoux? Comment se fait-il qu'elle tombe précisément sur Richard au moment où elle conduit la dépouille mortuaire de son mari ? Emrys Jones que l'on va consulter comme un oracle avoue comiquement qu'elle n'en sait rien. Pourquoi Richard cherche-t-il d'abord à éliminer Clarence? L'inquićtude de la reine est-elle fondée ? N'est-elle pas indûment hystérique ? Pourquoi faut-il écarter Hastings ? Autant de questions légitimes que posent les interprètes et qui sont autant de jalons de l'explication de textes historique, théâtrale qu'Al Pacino conduit soigneusement en prélude à l'interprétation des rôles. Parfois les mises en perspective sont éclairantes, les commentaires enrichissants : on rappelle utilement qu'Ann Warwick appartient au camp des vaincus, qu'elle a besoin de protection, qu'clle est fragilisée par la victoire des York, on souligne l'affection d'Hastings pour les jeunes princes, l'intimité des liens qui l'unissaient au défunt roi - ils 
partageaient la même femme, Mistress Shore. A d'autres moments, on a le sentiment qu'Al Pacino tâtonne et se fourvoie. Ainsi, lorsqu'il remplace le nom Clarence par le prénom George pour rendre la prophétie invoquée par Richard plus compréhensible pour un public contemporain, on n'est pas sûr qu'il perçoive l'ironie tragique du vers qui désigne l'autre «G», Gloucester, comme futur meurtrier (dans la pièce) des héritiers d'Édouard. Les réponses aux questions posées déconcertent parfois comme lorsque Pacino et Kimball décident de choisir une comédienne très jeune dans le rôle de Lady Ann pour rendre "plausible» la victoire de Richard alors que la scène tire toute sa force opaque el sa puissance dramatique de son invraisemblance même. Seul le démonisme du monstre claudiquant et sa sombre séduction rendent la scène théâtralement possible. Mais on comprend que pour le travail de l'acteur, seules comptent les hypothèses. La richesse et l'intensité de l'interprétation découlent de la radicalité et de la pertinence des questions, non de la justesse - théorique - des réponses.

Et de lait, la scène de la séduction de Lady Ann est une des plus convaincantes qu'il m'ait été donné de voir, au théâtre ou au cinéma. La fragilité de biche effarouchée de Winona Ryder, son étonnement gracile et innocent face aux avancées à la fois assurées et timides de Richard, qui, à chaque réplique, risque son va-tout, donnent à la scène, jouée tout en murmures et en intériorité une extraordinaire force émotionnelle. Emondée il est vrai de ses constructions rhétoriques les plus ostentatoires, réduite à quelques échanges, à une esquisse, la scène devient presque une scène d'amour où chacun des interprètes, à fleur de sensibilité, on voudrait dire à fleur d'âme, exprime saa vérité, dans un rapport dénué de tout artifice. Et l'on constate que si la magic du théâtre est là, le cinéma offre aux acteurs grâce aux gros plans cl à la possibilité de jouer à mi-voix, dans un souffle, d'exceptionnelles potentialités expressives. «Plus on parle bas, plus on est près des émotions» commente Peter Brook qui, loin de penser que le jeu cinéma est un artifice ou une trahison de la rhétorique dramatique, proclame les avantages que présente la proximité de la caméra pour le comédien. Le langage de Shakespeare, explique-t-il, c'est le langage de la pensée. Au théâtre, il faut projeter, et il y a peu d'acteurs qui savent à la fois parler fort et rester vrais. Au cinéma, si l'on dit les vers très calmement, sans timbrer, on ne va pas contre la nature du vers, on permet au contraire au vers d'être l'instrument d'expression d'un homme qui dit sa vérité intérieure. Plusieurs scènes bénéficient du surcroît d'émotion que permettent les gros plans et l'intimité du rapport à la caméra : la scène où Richard étreint Clarence et lui chuchote ses confidences à l'oreille (l'ironic tragique est décuplée par le langage de l'image), la scène de l'assassinat de Clarence dont les cadrages serrés, les voix murmurées accroissent l'intensité tragique des regards et des paroles, la scène de rupture entre Buckingham et Richard couronné où la méfiance et la mise à l'épreuve 
chez l'un, la soudaine compréhension des enjeux chez l'autre se lisent dans les yeux avant que les mots soient prononcés. Autant de scènes où l'exploration par la caméra met à jour des niveaux de conscience et des fulgurances de rapports émotionnels que ne permet pas le théâtre. Certes ces scènes sont à l'évidence écrites pour le théâtre mais l'expérimentation que permet le cinéma explore d'autres potentialités dramatiques, d'autrẹs résonances intéricures, présentes également dans le texte.

Ce sont toutes les scènes interprétées dans le film dont on admire la qualité de jeu, la puissance émotionnelle ou dramatique. Si Looking for Richard n'offre qu'un digest des grandes scènes de la pièce, on ne ressent pas ce choix comme limitatif car on n'a aucun mal à imaginer en pointillé une représentation complète de la pièce. Al Pacino a réuni là la distribution la plus étonnante dont on puisse rêver. De Penelope Allen (la reine Élisabeth) à Harris Yulin (le roi Édouard) en passant par Kevin Conway (Hastings) et Estelle Parsons (la reine Margaret), tous les comédiens sont remarquables de justesse et d'intensité : notamment Kevin Spacey en Buckingham chafouin et angoissé, Alec Baldwin en Clarence confiant et attendrissant, Penelope Allen en épouse et mère aux abois, craintive et véhémente. Certains rôles, d'habitude moins bien cernés, acquièrent un relief particulier que sculc leur confere la qualité de l'interprétation. C'est le cas des rôles du roi ct de la reine auxquels on ne prête guère attention dans d'autres mises en scène. Ici, Harris Yulin rend la maladie d'Édouard IV, qui colore la fin de l'Acte l et le début de l'Acte II et contamine tous les esprits, physiquement perceptible. Penelope Allen traduit de façon poignante et concrète les appréhensions et la lucidité politique de Lady Grey — la reine Élisabeth - que les autres membres du clan Woodville, aveugles, qualifient d'hystérique. Pour d'autres rôles, c'est peut-être notre mémoire cinématographique qui enrichit la perception. Si Kevin Spacey avec son regard enfantin nous convainc dans Buckingham et donne l'impression d'exister fortement en ne faisant rien, c'est en partic probablement parce que son rôle du boiteux anodin / Keyser Soze dans Usual Suspects et son rôle de serial killer psychopathe et obsessionnel dans Seven se superposent dans notre souvenir à son interprétation de Buckingham. Ce visage lisse qui inspire la confiance, cette apparence d'innocence nous glacent d'autant plus.

Il est certain aussi qu' Al Pacino dans Richard est lourd de tous les autres rôles de caïds et de mafiosi qu'il a interprétés au cinéma. Certains de ces rôles avaient déjà une dimension shakespearienne. «La filmographic pacinienne, écrit Serge Kaganski, est criblée de rôles qui auraient pu être écrits par le grand [Will] : le Corleone du Parrain est peut-être bien un Richard III faisant dans l'huile d'olive et la mitraille, le Montana de Scarface serait un fils putatif de Macbeth avec de la coke plein les naseaux et le Carlito de l'Impasse pourrait s'identifier au sort du roi Lear dépossédé de son pouvoir. Ainsi, les 
millions d'Américains qui on vu les films de Coppola ou de De Palma connaissent-ils un peu l'Élisabéthain, sans le savoir parce que des cinéastes bien intentionnés leur ont filé un peu de Shakespeare en contrebande» ${ }^{15}$.

La qualité majeure de l'acteur qui joue Richard au théâtre ou au cinéma est à l'évidence la capacité protéenne de métamorphose. Tour à tour fraternel et tendre avec Clarence, chevaleresque et séducteur avec Lady Ann, affectueux et presque matcrnel avec les enfants d'Édouard, dévôt et patelin avec le lord Maire de Londres, confiant et proche puis irritable et glaçant avec Buckingham, Richard est l'acteur par excellence, qui déploie avec virtuosité toutc une palette de rôles et de masques. La duplicité est son génie et à plusicurs reprises il compare lui-même l'art de la (dis)simulation à l'art du comédien :

And thus I clothe my naked villainy,<smiles>C1[AlH]C[AlH]1</smiles>

And seem a saint when most I play the devil.

ou cncore, dialoguant avec Buckingham :

Rich. Come, cousin, canst thou quake and change thy colour, Murder thy breath in middle of a word. And then again begin, and stop again. As if you were distraught and mad with terror?

Buck. Tut, I can counterfeit the deep tragedian, Speak, and look back, and pry on every side, Tremble and start at wagging of a straw Intending deep suspicion. Ghastly looks Are at my service like enforced smiles And both are ready in their offices At any time to grace my stratagems.

(Richard III, III.5.1-11) ${ }^{17}$

Tout l'art du comédien qui interprète le rôle de Richard doit être de fasciner et de séduire pour que ce double jeu ne soit guère plus déchiffrable par le spectateur qu'il ne l'est par les personnages de la pièce ${ }^{18}$. A ce jeu, Pacino démontre avec éclat qu'il est l'un des meilleurs: se transformant physiquement au cours du film, tour à tour énormément cabotin ou retenu à l'extrême, il irradie, comme Richard, du bonheur de jouer. Il cabotine, s'adresse à la caméra, supplie : «Arrêtez-moi !». Et puis, l'œil en maraude, de sa voix de gorge graillonnante, cette même voix qui plaida pour Michel Corleone ou Tony Montana, il lâche au ciel un rire de fou qui n'appartient qu'à Richard III. 
Dans ce rôle, il s'impose avec une économie de moyens admirable. Très souvent, il le proclame dans les interviews, il semble ne pas jouer mais derrière le masque, l'apparente candeur du regard, le mutisme opiniâtre, le visage fermé, que de bouillonnements! Tour à tour fiévreux, fou, glaçant, terrible (Ah ! le «If» de la scène des fraises où il foudroie d'un scul mot le pitoyable Hastings !) il est magnifique. Jusqu'à la scène finale («Mon royaume pour un cheval !») filmée d'abord avec un filtre coloré puis en couleurs naturelles dans les champs où, traqué comme un sanglier. Richard s'effondre en luttant jusqu'au bout. Scène finale qu'à son habitude Al Pacino désamorce en nous mimant sur les marches d'un escalier une mort hoquetante et facétieuse. Tout le charme du film est là. Dans cet humour qui est la politesse du sérieux et de l'engagement.

De ce Richard III à la fois prismatique et impénétrable, quelle serait par rapport à ses devanciers au cinéma, l'originalité de la composition? Le critique du Sunday Times suggère une analyse comparative assez bien vue : «Pacino, écrit-il, delves into Richard and comes up with something fresh, despite the competition. Olivier played the role with lipsmacking relish, amazed at his own bad deeds. [...] Ian McKellen played him thin-lipped with disdain, amazed that goodness lets him get away with those deeds; interpretation of the role would seem to be strung out between these two poles - the vitality of evil or the banality of evil. But Pacino's black, basilisk stare bores through into something we'd all but forgotten : how about the plain old evilness of evil ?»19. Le mystère, la malignité, l'opacité indéchiffrable du mal, voilà le choix interprétatif majeur que par sa dominante d'impassibilité semble faire Al Pacino. Laissons à ce journaliste le mot de la fin. Car il y aurait encore beaucoup à dire pour rendre justice à ce film foisonnant, hymne jubilatoire au théâtre et aux comédiens. Looking for Richard et ses une heure cinquante-quatre de bonheur sont susceptibles, me semble-t-il, de réjouir le néophyte autant que le spécialiste, le shakespearien passionné autant que le spectateur anonyme fidèle à l'interprète ćblouissant du Parrain ou de L'épouvantail, sans parler du plus récent Associé du diable où Al Pacino est, à la lettre, diaboliquement diabolique.

Jean-Michel DÉPR ATS Université de Paris X - Nanterre 


\section{N O T E S}

${ }^{1}$ Chiffrc fourni par le mensuel Première, n²51, février 1998, p. 18.

2 Qu'on pourrait sculement comparer au Filming Othello d'Orson Welles (1978).

"Looking for Richard, États-Unis, 1996, Ih54. Réal. : Al Pacino, d'après Richard III de William Shakespeare. Commentaire écrit par Al Pacino et Frederic Kimball. Dir. photo: Robert Leacock, Nina Kedrem, John Kranhouse, Steve Confer. Dir. art. : Kevin Ritter. Cost.: Aude Bronson-Howard, Deborah Scott, Yvonne Blake. Mont. : Pasquale Buba, William A. Anderson, André Betz, Ned Bastille. Mus. : Howard Shore. Prod. : Michael Hadge et Al Pacino. Prod. exéc. : William Teitler. Prod. assoc. : James Bulleit. Cie de prod. : Twentieth CenturyFox. Dist. fr.: UFD. Int. : Penelope Allen (la reine Elisabeth), Alec Baldwin (Clarence), Kevin Conway (Hastings), Al Pacino (Richard III), Estelle Parsons (la reine Margaret), Aidan Quinn (Richmond), Winona Ryder (Lady Ann), Kevin Spaccy (Buckingham), Harris Yulin (le roi Édouard).

4 Dans l'ouvrage publié par les Presses de l'UFR CLERC de l'Université de Picarlic, Le tyran, Shakespeare contre Richard III (collection Sterne, éd. D. GoyBlanquet, Amiens, 1990), Fortunato Israël ne dénombre pas moins de dix mises en scène de Richard III sur la scène française depuis le début du siècle. Il faudrait y ajouter. depuis, la mise en scène iconoclaste et inspirée de Matthias Langhoff avec Marcial di Fonzo Bo (Festival d'Avignon, 1995). Dans ce même ouvrage, Lois Potter évoque el analyse de façon extrêmement vivante les interprétations d'Anthony Sher et de Ian McKellen (The actor as regicide, Recent versions of Richard III on the English stage).

5 Pour fairc contrepoids à ce jugement assurément sévère, on peut lire l'éloge qu'Ann Lecercle fait du jeu d'Olivier dans Shakespeare et le corps à la Renaissance (Société Française Shakespeare, Actes du Congrès 1990, Paris, les Belles Lettres, 1991) p. 21.3-215.

${ }_{7}^{6}$ Propos rapportés dans Les Inrockuptibles n89 (29/1 au 4/2/97).

7 lbid., p. 23.

${ }^{8}$ Dans son compte-rendu extrêmement riche paru dans le même numéro d'Inrockuptibles sous le titre: «Un roi à New York».

Première, $\mathrm{n}^{\circ} 251$. Interview avec Éric Libiot, p. 60.

${ }^{10}$ The quest: The play ; Casting the actors ; The birthplace of Shakespeare ; (jetting in deeper; Lady Ann; The murderers; Now to take the crown; Buckingham : Hastings ; The council meeting ; Richard is king ; The last act ; Richmond; The battle.

11 Ibid., p. 22.

12 Dite par Heathcote Williams, ami d'Al Pacino et dramaturge anglais dont Al Pacino avait adapté une pièce de théâtre pour tourner il y a dix ans un premier film de 52 minutes intitulé The Local Stigmatic. Cf. The Guardian, 3/1/1997, p. 3.

${ }^{13}$ Des rois dans la tempête, film de Georges Banu, Jean-Michel Déprats, 
Claude Mouriéras, diffusé le 22 avril 1997 sur Arte.

14 «Vanessa Redgrave's comment $[. .$.$] is gibberish of such pure, unadorned$ nature it makes you weep tears of shame that any American actor ever felt scared off the pitch». Tom Shone, "In search of Shakespeare's worst villain», Sunday' Times Culture, 2/2/97.

${ }^{15}$ Les Inrockuptibles, ibid., p. 23.

16 Richard III, édition Arden, I.3.336-338, p. 169-70.

17 Op. cit., p. $237-8$.

18 Sur l'histrionisme de Richard, voir, entre autres, A.P. Rossiter, Ange' with horns, 1961, p. 15 sq. et Wolfgang Clemen, A commentary on Shakespecure's Richard III, passim (p. 62, 139, 140, 144, 146, 148).

${ }^{19}$ Sunday Times du 2/2/1997. 